\title{
Experiments on Synchronization and Control of Chaos on Coupled Electrochemical Oscillators
}

\author{
István Z. Kiss, ${ }^{\dagger, *}$ Vilmos Gáspár,,${ }^{\dagger}$ and John L. Hudson*,* \\ Institute of Physical Chemistry, University of Debrecen, H-4010 Debrecen, P.O. Box 7, Hungary, \\ and Department of Chemical Engineering, Thornton Hall, University of Virginia, \\ Charlottesville, Virginia 22903-2442
}

Received: February 21, 2000; In Final Form: May 3, 2000

\begin{abstract}
Experiments were carried out on synchronization and control of complex chaotic dynamics observed during the dissolution of two and four coupled nickel electrodes in sulfuric acid under potentiostatic conditions. In a given potential range the individually measured currents exhibit asynchronous chaotic oscillations. The complexity (as measured by the correlation dimension) of the chaotic oscillations depends on the extent of coupling among the electrodes. Thus, the effectiveness of a combined synchronization-delayed-feedback procedure can be tested on systems with increasing complexity. We show that the asynchronous chaotic oscillations can be converted to synchronized and simple, periodic current oscillations by a two-step procedure. The chaotic current oscillations of coupled electrodes are first synchronized by perturbations of external resistors that are connected individually to each electrode. Then, the desired periodic orbit is stabilized by perturbations of the potential. We also observed that certain nonvanishing perturbations could lead to only partially synchronized, so-called "clustered" chaotic states.
\end{abstract}

\section{Introduction}

Control and synchronization of chaotic systems have been the subject of intense research during the past decade. A comprehensive review on the topic can be found in a recently published special issue of Chaos. ${ }^{1}$

Control of low-dimensional chaotic systems has been studied both theoretically and experimentally. The goal is typically the stabilization of unstable periodic orbits embedded in a chaotic time signal. Several algorithms have been developed and tested in different experimental settings. For example, chaos in the Belousov-Zhabotinsky (BZ) ${ }^{2}$ reaction was controlled by a very simple, proportional feedback $(\mathrm{SPF})^{3}$ algorithm. The Pyragas method $^{4}$ was successfully applied to the $\mathrm{BZ}^{5}$ and an enzyme reaction. ${ }^{6}$ Simple and recursive proportional feedback ${ }^{7}$ methods, adaptive learning algorithm, ${ }^{8}$ and the Pyragas method were successfully applied to electrochemical systems. ${ }^{8-11}$ Small, periodic modulation of a control parameter has been applied to stabilize periodic orbits in the chaotic BZ system. ${ }^{12}$ In all these experiments, the chaotic systems were locked onto periodic limit cycles. Recently, derivative ${ }^{13}$ and time-delay ${ }^{11}$ control methods were successfully applied to stabilize the system on originally unstable steady states as well.

In spatiotemporal systems, however, interactions among the sites have to be considered. ${ }^{14-16}$ Control and synchronization procedures have been employed in coupled map lattices (CML's), ${ }^{17-19}$ coupled chaotic oscillators, ${ }^{20,21}$ and partial differential equations. ${ }^{22}$ Some of these results have been recently reviewed..$^{23-25}$ Synchronization of two chaotic chemical systems has been carried out in numerical studies. ${ }^{26-28}$ Spatially extended chemical systems were also synchronized. ${ }^{24,29}$

* Authors for correspondence.

¿ University of Debrecen.

$\doteqdot$ University of Virginia.
High-dimensional spatiotemporal chaotic systems are known in electrochemistry. For example, complex chaotic current oscillations during the anodic electrodissolution of an iron disk in sulfuric acid electrolyte have been observed. ${ }^{30}$ Electrode arrays $^{31,32}$ have been used to study spatiotemporal chaotic electrochemical processes. The array consists of a number of small disks (free ends of wires embedded in an insulator). It can be regarded as a physically realistic implementation of CML's. The coupling between the sites is the result of the complex physical (migration, diffusion) and chemical (dissolution, film formation, gas evolution) processes. As the coupling between the sites is mainly through the electrolyte, the array can be considered as a rough model of an electrode of the same size. Indeed, excellent agreement has been found between activation and passivation waves on arrays ${ }^{31}$ and a disk. ${ }^{33}$

It should be noted that coupling of electrochemical oscillators through external circuitry including resistors, capacitors, and inductors was investigated by Franck and Meunier some time ago. ${ }^{34}$ They showed how phase locking of periodic oscillators is produced by means of the coupling.

In recent publications we have investigated ${ }^{35-37}$ the spatiotemporal chaotic behavior of globally coupled arrays of nickel electrodes. In this paper, we now report on synchronization and control of the chaotic dynamics using feedback methods. We investigate four cases: two- and four-electrode arrays with no and some added constant global coupling. Without feedback control, the dynamics become more complex as the number of elements is increased or, alternatively, as the extent of global coupling is decreased. We thus investigate the effect of control on four base cases that have varying chaotic complexity and spatial heterogeneity. 


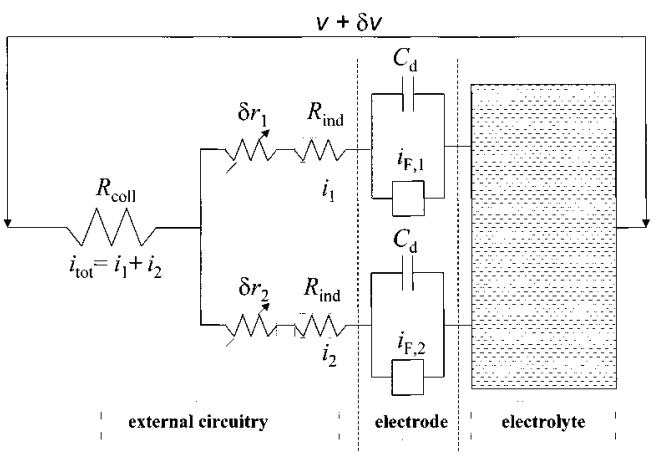

Figure 1. Equivalent circuit of the cell with two electrodes, where $v$ is circuit potential, $C_{\mathrm{d}}$ is double-layer capacitance, $R_{\text {coll }}$ is collective resistance, $R_{\text {ind }}$ is individual resistance, $i_{\text {tot }}$ is total current, $i_{k}$ and $i_{\mathrm{F}, k}$ are the currents and the Faradaic currents of the electrodes $(k=1,2)$, respectively. Perturbations to the circuit potential and to the individual resistances are denoted by $\delta v$ and by $\delta r_{k}$ respectively.

\section{Experimental Section}

The experimental details are given in an earlier study. ${ }^{36}$ An array of $\mathrm{Ni}$ electrodes is applied as the working electrode in a standard three-electrode cell. The circuit potential is held constant with respect to a $\mathrm{Hg} / \mathrm{Hg}_{2} \mathrm{SO}_{4} / \mathrm{K}_{2} \mathrm{SO}_{4}$ reference electrode by a potentiostat (model 273, EG\&G Princeton Applied Research). The counter electrode is a Pt sheet. The electrolyte is $4.5 \mathrm{~mol} \mathrm{dm}^{-3} \mathrm{H}_{2} \mathrm{SO}_{4}$ solution. The electrodes are connected to the potentiostat through collective and individual resistors. Small perturbations of the individual resistors (frequency 40 $\mathrm{Hz}$, accuracy $\pm 1.17 \Omega$ ) and the circuit potential (frequency 200 $\mathrm{Hz}$, accuracy $\pm 0.01 \mathrm{mV}$ ) are carried out, respectively, by a computer-controlled resistor box (EF-499, Elektroflex, Hungary) and an 8-bit D/A card. The local currents flowing through each electrode were measured with zero-resistance ammeters. Control experiments were carried out with two or four electrodes in a $2 \times 2$ geometry.

Chaotic Behavior of Uncontrolled Coupled Electrodes. In previous studies, ${ }^{35,37}$ we investigated the effect of global coupling on the chaotic dynamics of interacting electrodes. The coupling can be changed while holding all other parameters constant. This is done through the use of collective $\left(R_{\text {coll }}\right)$ and individual $\left(R_{\text {ind }}\right)$ resistors (Figure 1); the total external resistance $\left(R_{\text {tot }}\right)$ is held constant while the fraction dedicated to individual currents, as opposed to the total current, is varied.

The resistance value $R_{\text {tot }}$ that gives the total resistance of the circuit is defined as follows:

$$
R_{\mathrm{tot}}=R_{\mathrm{coll}}+\frac{R_{\mathrm{ind}}}{n}
$$

where $n$ is the number of electrodes. As in our previous study, ${ }^{36}$ we define an equivalent resistance $R_{\text {eq }}=n R_{\text {tot }}$, which is held constant $\left(R_{\text {eq }}=200 \Omega\right)$ as $n$ is changed. Increasing the collective resistance (while keeping both $n$ and $R_{\text {tot }}$ fixed) results in a stronger global coupling. The extent of global coupling is then given by the ratio

$$
\epsilon=R_{\mathrm{coll}} / R_{\mathrm{tot}}
$$

The value of $\epsilon$ can be varied between 0 (no global coupling) and 1 (maximum global coupling). The effect of $\epsilon$ on the complexity of the individual and total currents was thoroughly studied in a previous publication. ${ }^{35}$ The complexity of these signals was characterized by the information dimension of the chaotic attractors reconstructed from the corresponding time series data. It was shown that by increasing the coupling the
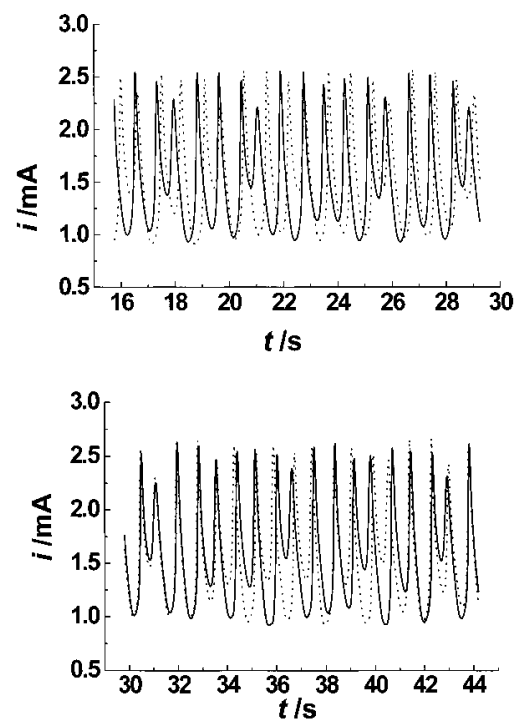

Figure 2. Chaotic time series of local currents for a two-electrode setup. Solid line, electrode 1; dotted line, electrode 2. (a, top) $V=$ $1.330 \mathrm{~V}, \epsilon=0, R_{\text {eq }}=200 \Omega$, (b, bottom) $V=1.340 \mathrm{~V}, \epsilon=0.2, R_{\text {eq }}$ $=200 \Omega$.

complexity of the total current decreases while that of the individual current goes through a maximum.

In the present work we consider two representative cases of coupling in both two-electrode and four-electrode configurations:

(a) No added global coupling $(\epsilon=0)$ : maximal complexity in the total current. There is no added coupling through collective resistor. Without added global coupling the individual chaotic elements oscillate almost independently. There is always some long-range coupling among the electrodes through the electrolyte. The resistance of the electrolyte is small $(2-3 \mathrm{ohms})$ which is much less than the resistance in the external circuit. As we pointed out earlier, ${ }^{36}$ the coupling through the electrolyte can be neglected in describing the dynamics.

(b) Added global coupling: maximal complexity in the individual currents $(\epsilon=0.2$ for $n=2$ and $\epsilon=0.4$ for $n=4)$. ${ }^{35}$

In Figures 2 and 3 we show the effect of adding global coupling to the sets of two and four electrodes, respectively. In Figure 2, time series data are presented for the two-electrode set for no global coupling (a) and some added coupling (b). Without coupling, the electrodes oscillate almost independently. The added coupling brings the time series closer together. The two signals are almost in phase but the amplitudes still vary significantly. We use the correlation dimension ${ }^{38}$ to help characterize the signals. The dimensions of the attractors reconstructed from the total currents corresponding to Figure 2 , a and b, are 3.5 and 2.8 , respectively.

Some behavior on the four-electrode array without and with some added global coupling is shown in Figure 3. Without added coupling, Figure $3 \mathrm{a}$, the elements oscillate almost independently. The dimension of the attractor reconstructed from the total current is large; the method used yields a value of 4.8 but we note that estimates of such large values are approximate. With added coupling, Figure 3b, the dimension calculated from the total current is considerably lower (2.9).

We shall use the four types of behavior shown in Figures 2 and 3 as testbeds for the application of synchronization and chaos control methods.

Synchronization and Control Procedures. We shall apply a two-step procedure for synchronization and control of the spatiotemporal system. We use as an observable variable the 

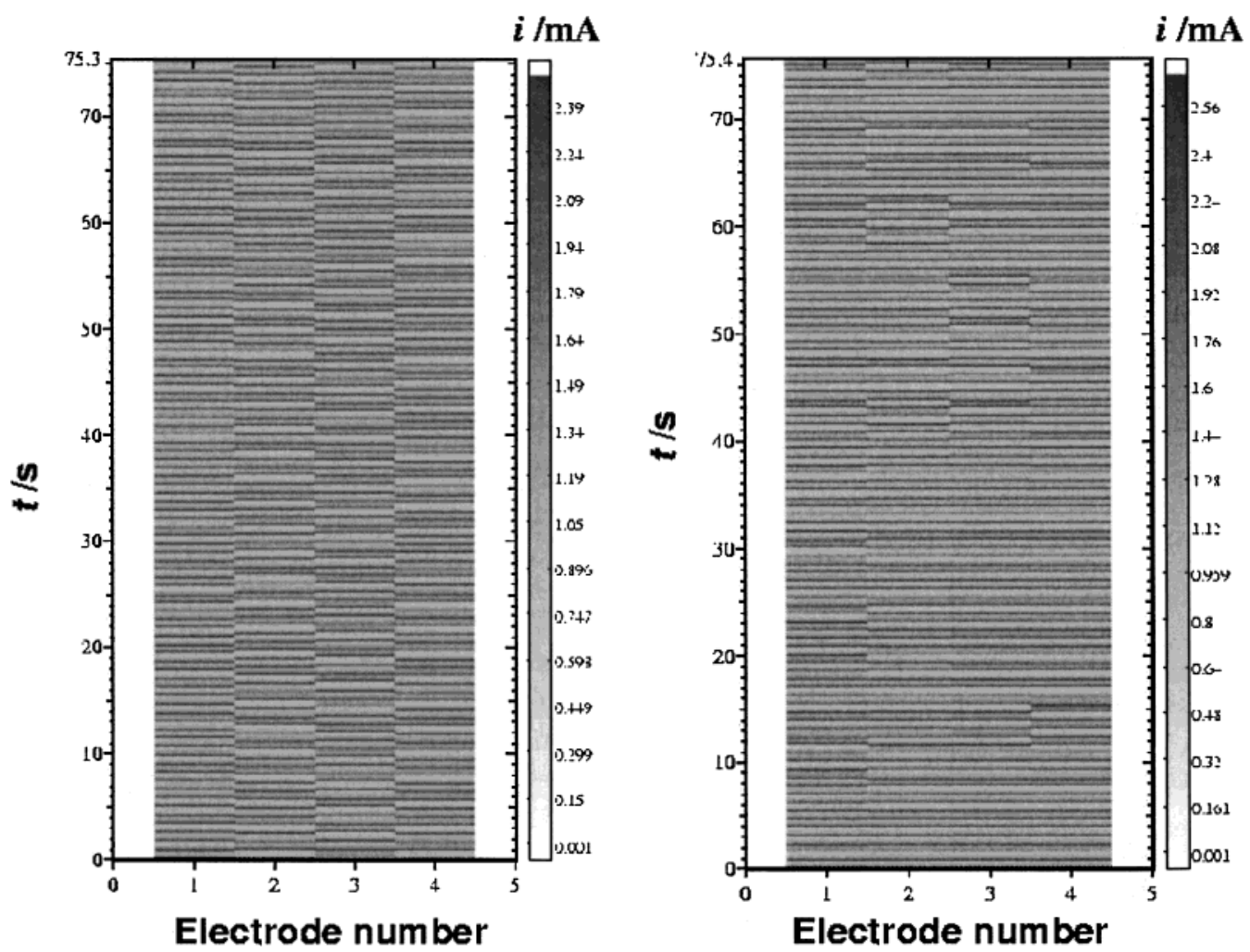

Figure 3. Grayscale plot of chaotic local currents of the four-electrode setup. $V=1.320 \mathrm{~V}, R_{\mathrm{eq}}=200 \Omega$. (a, left) $\epsilon=0$, and (b, right) $\epsilon=0.4$.

individual current $i_{k}(t)$ of each element $k=1, \ldots, n$. The total current

$$
i_{\text {tot }}(t)=\sum_{k=1}^{n} i_{k}(t)
$$

then characterizes the global dynamics of the system.

An experimentally accessible system parameter $r_{k}$ (the external resistor on each of the individual electrodes) is available that affects the local dynamics of the subsystem. Synchronization of the subsystems can be achieved by simultaneously perturbing each $r_{k}$ according to

$$
\delta r_{k}(t)=K\left(i_{k}(t)-\frac{i_{\text {tot }}(t)}{n}\right)
$$

where $K$ is a coupling constant. When synchronization is successful, $i_{k}(t)=i_{\mathrm{tot}}(t) / n$ for all $k$, and the local perturbations vanish. To avoid "explosion" of the dynamics, we set an upper limit $\delta r_{\max }$ to the perturbations such that if $\left|\delta r_{k}(t)\right| \geq \delta r_{\max }$ then only $\delta r_{\max }$ is applied with the correct sign.

Once the system is synchronized, a simple delayed-feedback formula ${ }^{4}$ is applied to stabilize the desired unstable periodic orbit of period $\tau$ by continuous perturbations (see eq 5) of a global parameter $v$ (which in our work is the circuit potential):

$$
\delta v(t)=C\left[i_{\mathrm{tot}}(t)-i_{\mathrm{tot}}(t-\tau)\right]
$$

where $C$ is the control constant. The perturbation $\delta v(t)$ is also limited to $\delta v_{\max }$.

The success of the two-step strategy greatly depends on properly choosing the values for $n, K, C$, and $\tau$. In the present study, the algorithm was applied to $n=2$ and 4 only because of the limitations in the experimental setup. We have extensively tested for the effectiveness of synchronization by varying the value of coupling constant $K$, while the best value of control
TABLE 1: Procedures To Stabilize a Uniform Periodic State for the Four Studied Systems of Different Complexities $^{a}$

\begin{tabular}{cccccc}
\hline & & \multicolumn{3}{c}{ procedures to stabilize the unstable P2 orbit ${ }^{b}$} \\
\cline { 3 - 6 }$n$ & $\epsilon$ & $D_{2}$ & $\begin{array}{c}\text { synchronizn } \\
\text { for targeting }\end{array}$ & $\begin{array}{c}\text { synchronizn } \\
\text { for control }\end{array}$ & $\begin{array}{c}\text { delayed feedback } \\
\text { method }\end{array}$ \\
\hline 4 & 0 & 4.8 & + & + & + \\
2 & 0 & 3.5 & + & + & + \\
4 & 0.4 & 2.9 & + & - & + \\
2 & 0.2 & 2.8 & - & - & +
\end{tabular}

${ }^{a} n=$ no. of electrodes. $\epsilon=$ global coupling. $D_{2}=$ correlation dimension. ${ }^{b}+$ required, - not required.

constant $C$ was found by trial and error. The period $\tau$ was estimated from the time series of the unperturbed systems.

\section{Results}

The four test systems to which we apply synchronization and/ or "chaos" control, are summarized in Table 1. The systems are listed in a decreasing order of complexity: $(n=4, \epsilon=0)$, $(n=2, \epsilon=0),(n=4, \epsilon=0.4)$, and $(n=2, \epsilon=0.2)$. The procedures (in addition to the delayed feedback method) required to bring the systems onto synchronized periodic behavior varies among the four cases.

Synchronization of Chaotic Elements. We begin with a discussion of procedures applied to the test systems with the goal of synchronization into a uniform chaotic state.

Figure 4 shows the synchronization of two noncoupled $(\epsilon=$ 0 ) chaotic electrodes. The synchronization procedure (using eq 4) was applied in the middle region, approximately between $t$ $=26$ and $t=77 \mathrm{~s}$. Note that synchronization results in a small increase in the amplitude of the chaotic total current (Figure $4 a)$, while the difference between the chaotic local currents becomes negligible (Figure 4b). Synchronization requires continuous perturbations of the individual resistance as that shown for one of the electrodes in Figure 4c. However, the 

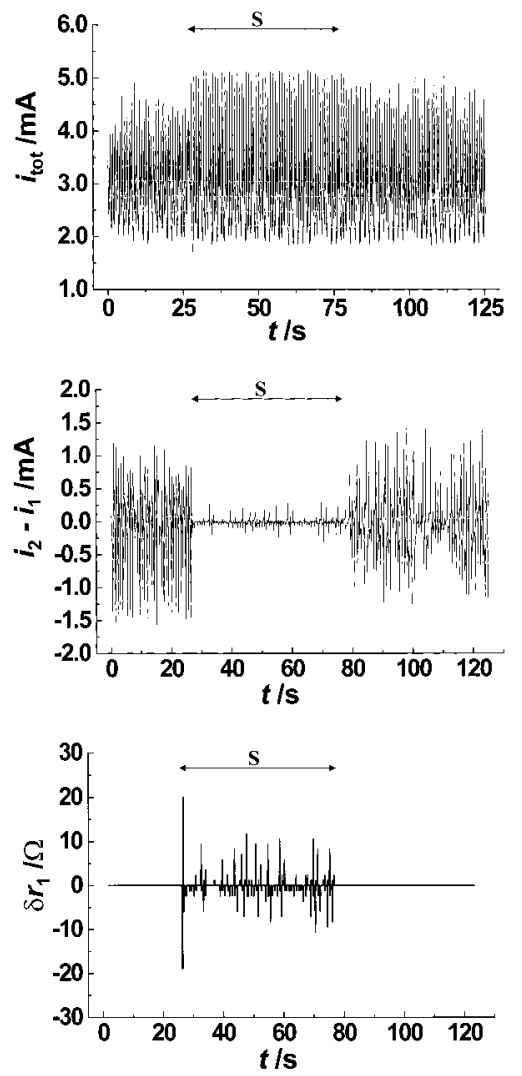

Figure 4. Synchronization of two electrodes at $\epsilon=0$ (for the other experimental conditions see Figure 2a). Synchronization was turned on between $t=26.13$ and $76.675 \mathrm{~s}($ period $S)$ with $K=-80 \Omega \mathrm{mA}^{-1}$ in eq $4\left(\delta r_{\max }=20 \Omega\right)$. (a, top) Time series of the total current, (b, middle) difference between the individual currents, (c, bottom) perturbations to the individual resistors connected to electrode 1 .

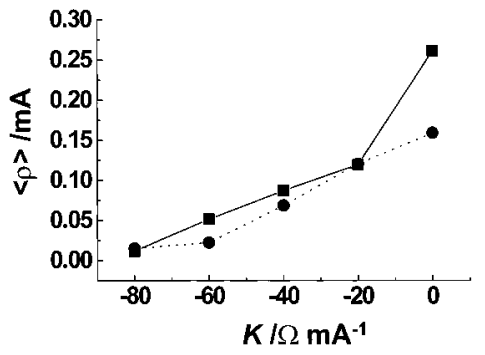

Figure 5. Extent of synchronization $\langle\rho\rangle$ as a function of coupling strength $K$ in the case of two electrodes. Solid curve: $\epsilon=0$, dashed curve: $\epsilon=0.2$. Other data are as in Figure 2.

perturbations are small, an order of magnitude less than the individual resistors $(200 \Omega)$ applied in the given circuit. When synchronization is turned off, the system becomes unsynchronized again. The results shown in Figure 4 were obtained with a value of the constant $K$ of $-80 \Omega \mathrm{mA}^{-1}$. The electrodes will not synchronize if $|K|<80 \Omega \mathrm{mA}^{-1}$.

To quantify the extent of synchronization, we calculate $\rho(t)$, which is a measure of the size of the current distribution:

$$
\rho(t)=-\frac{1}{n_{k=1}^{n}}\left|i_{k}(t)-i_{\mathrm{tot}}(t) / n\right|
$$

Note that $\rho(t)$ is identically zero for synchronized elements. We then take an average $\rho(t)$ for a time $T$ :

$$
\langle\rho\rangle=\frac{1}{T} \int_{t=0}^{T} \rho(t) \mathrm{d} t
$$
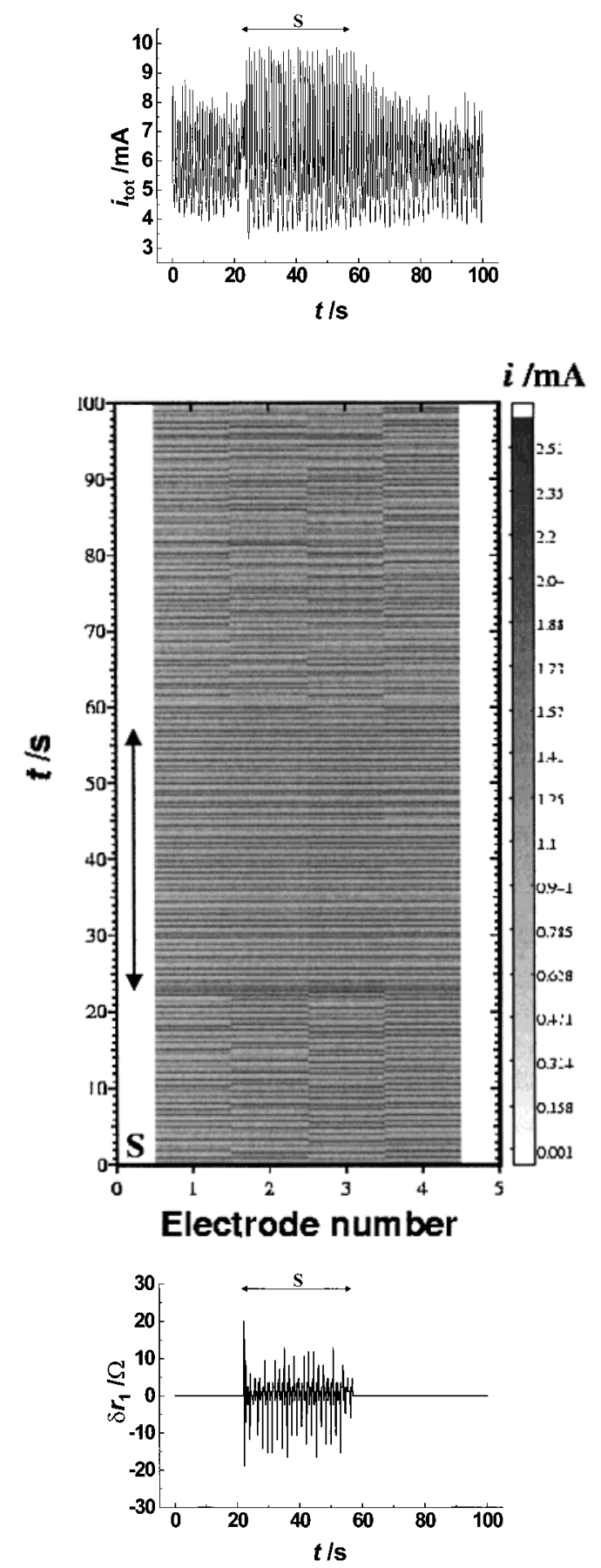

Figure 6. Synchronization of four electrodes at $\epsilon=0$ (other conditions are as in Figure 3). Synchronization was turned on between $t=22.14$ and $57.115 \mathrm{~s}$ (period $S$ ) with $K=-80 \Omega \mathrm{mA}^{-1}$ in eq $4\left(\delta r_{\max }=20\right.$ $\Omega$ ). (a, top) Time series of the total current, (b, middle) grayscale plot of the individual currents, (c, bottom) synchronizing perturbations applied to electrode 1 .

Figure 5 shows the dependence of $\langle\rho\rangle$ on the value of coupling constant $K$ for two noncoupled electrodes $(\epsilon=0)$. The electrodes become more and more synchronized as the strength of perturbations is increased and synchronization is achieved at $K$ $=-80 \Omega \mathrm{mA}^{-1}$.

Also shown in Figure 5 is a result obtained for the twoelectrode configuration with added global coupling $(\epsilon=0.2)$; without synchronization $\left(K=0 \Omega \mathrm{mA}^{-1}\right)$ the value of $\langle\rho\rangle$ is significantly less than that obtained for $\epsilon=0$. For $\epsilon=0.2$ the value of $\langle\rho\rangle$ decreases with increasing $|K|$ until synchronization is obtained; this occurs when $K=-60 \Omega \mathrm{mA}^{-1}$, i.e., at a 


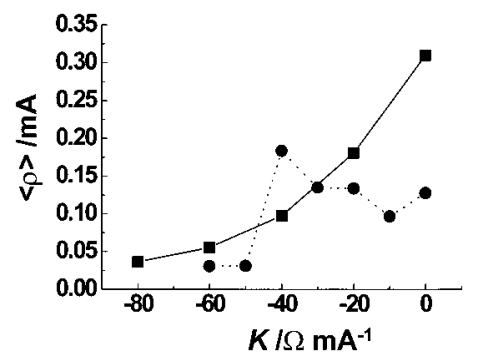

Figure 7. Extent of synchronization $\langle\rho\rangle$ as a function of coupling strength $K$ in the case of four electrodes. Solid curve: $\epsilon=0$, dashed curve: $\epsilon=0.4$. Other conditions are as in Figure 3.

slightly smaller absolute value of the constant $K$ than that needed when $\epsilon=0$.

Similar experiments have been carried out with a fourelectrode setup. Figure $6 \mathrm{a}-\mathrm{c}$ shows synchronization of chaotic oscillations of four noncoupled electrodes $(\epsilon=0)$. Again, synchronization results in an increase in the amplitude of the chaotic total current (Figure 6a). The synchronization, shown by a grayscale plot of the individual currents in Figure 6b, requires the continuous perturbations of the individual resistance values. Note in Figure $6 \mathrm{c}$ that the perturbations are a bit larger than those applied earlier at the two-electrode setup. When synchronization is turned off, the system becomes unsynchronized again (Figure 6b).

The $\langle\rho\rangle$ versus $K$ plot for the four-electrode setup is shown in Figure 7. For noncoupled electrodes $(\epsilon=0)$ the curve is similar to that observed for two noncoupled electrodes. However, for coupled electrodes $(\epsilon=0.4)$ a maximum has been found at $K=-40 \Omega \mathrm{mA}^{-1}$. The grayscale plot of the individual currents (Figure $8 \mathrm{a}$ ) reveals that at this value of coupling constant the electrodes form two groups (clusters) and the system is only partially synchronized: electrodes 1,2 , and 4 oscillate in the same phase, while electrode 3 is out-of-phase. Note in Figure $8 b, c$ that, perhaps due to the partial synchronization, both the total and local currents oscillate in a less chaotic fashion.

Controlling Chaos. Once the chaotic electrodes are synchronized, the continuous delayed-feedback algorithm that was described above can be applied to stabilize the desired unstable periodic orbit through perturbations of the applied potential.

Figure 9a,b shows control of period-2 current oscillations of two and four noncoupled electrodes, respectively. The spacetime plot of the four-electrode experiment is shown in Figure 10. When control is turned off, the system returns to the synchronized chaotic state. After turning off the synchronization as well, the original asynchronous chaotic behavior is recovered. We have observed that if synchronization was turned off while control was still on, the chaotic behavior reappeared after few oscillations. This finding indicates that in these high-dimensional chaotic systems $\left(D_{2}>3\right)$ both local and global perturbations are necessary to achieve successful chaos control.

On the other hand, in the case of four coupled electrodes $(\epsilon$ $=0.4, D_{2}=2.9$ ), which constitutes a less complex chaotic system, the periodic oscillations remained stable even if synchronization was turned off while control was on (Figure 9c). However, without a preceding synchronization session, chaos control could not be achieved. Therefore, in this case, synchronization acts as a targeting procedure, and when the system is in the neighborhood of the periodic orbit the delayed feedback algorithm stabilizes the periodic oscillations.

In the case of the two coupled electrodes $(\epsilon=0.2)$, synchronization was not necessary to stabilize the uniform periodic oscillations (Figure 9d). In contrast to the other, more
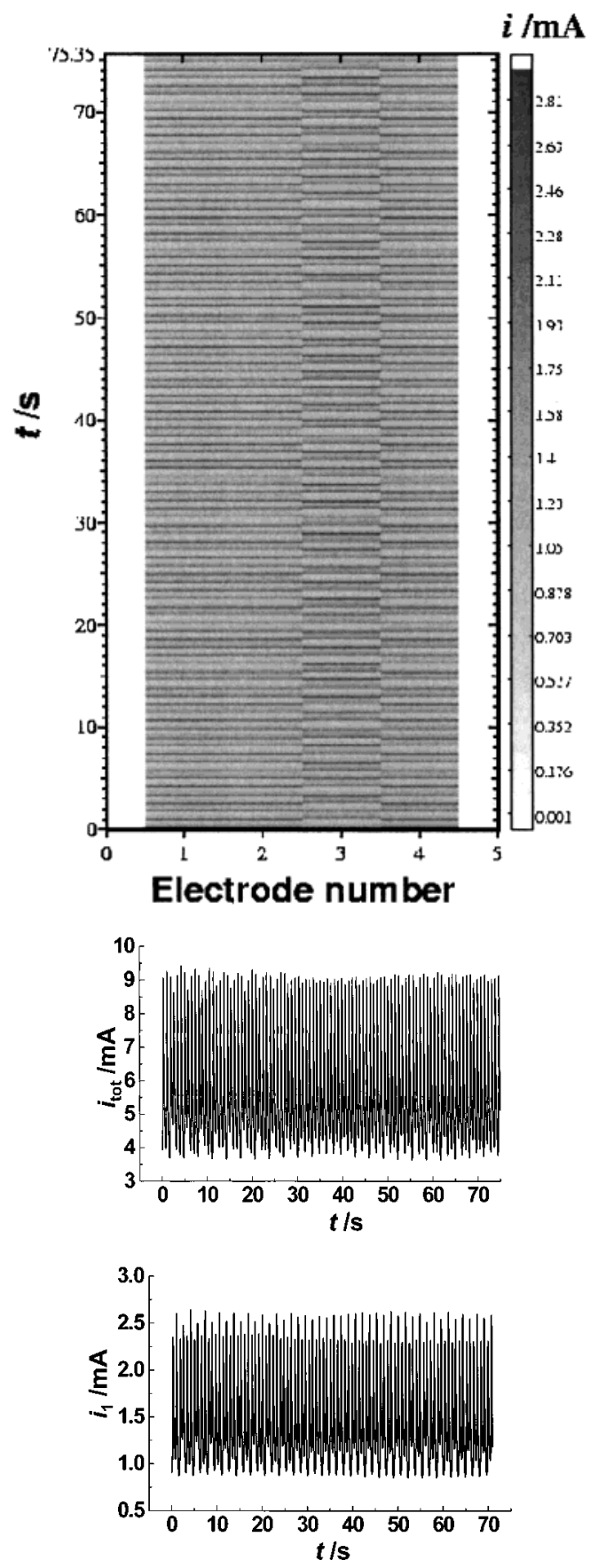

Figure 8. Partial synchronization (cluster formation) in the fourelectrode setup at $\epsilon=0.4$ (other data are as in Figure 3). The individual resistors are continuously perturbed according to eq 4 with $K=-40$ $\Omega \mathrm{mA}^{-1}\left(\delta r_{\max }=20 \Omega\right)$. (a, top) Grayscale plot of the individual currents, (b, middle) time series of total current, (c, bottom) time series of the current of electrode 1 .

complex states (Figure 9a-c), the simple delayed-feedback applied to the circuit potential is enough to produce a successful control.

The results of the control experiments are summarized in Table 1. For the two most complex systems $(\epsilon=0)$, both synchronization and the delayed-feedback methods were required for successful stabilization of the periodic orbit. For an intermediate case $(n=4, \epsilon=0.4)$ synchronization was required only in the introductory phase of the control procedure: it was not needed in maintaining successful control. In the case of the simplest system $(n=2, \epsilon=0.2)$, the delayed-feedback method alone was capable of stabilizing the unstable periodic orbit. 

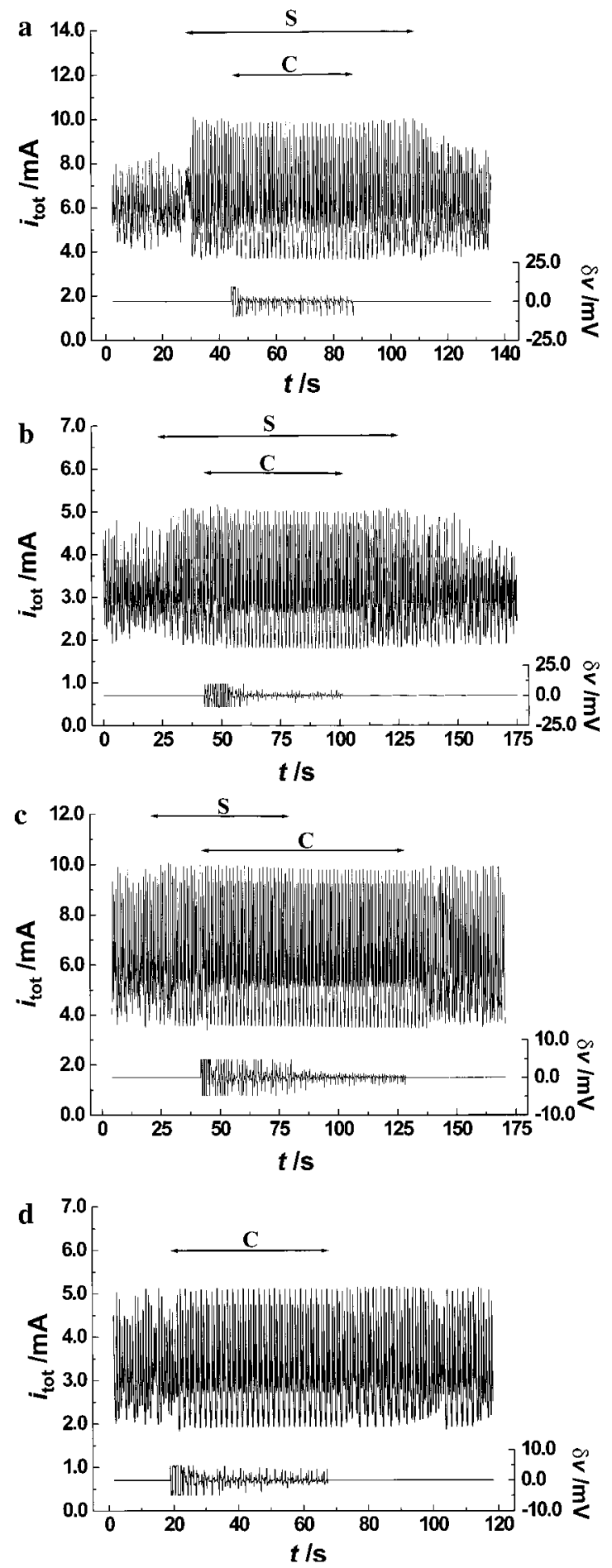

Figure 9. Time series of the total current (left axis) and perturbations of the circuit potential (right axis) during stabilization of period-2 oscillations. The individual resistors are perturbed according to eq 4 during a period of length $S$, while the circuit potential is perturbed according to eq 5 during a period of length $C$ as shown in the figures. (a) Four electrodes. $\epsilon=0$, (other data are as in Figure 3a). During session $S: K=-100 \Omega \mathrm{mA}^{-1}$ and $\delta r_{\max }=20 \Omega$. During session $C$ : $\tau=1.52 \mathrm{~s}, C=0.00956 \mathrm{~V} \mathrm{~mA}^{-1}$, and $\delta v_{\max }=10 \mathrm{mV}$. (b) Two electrodes. $\epsilon=0$ (other data are as in Figure 2a). During session $S: K$ $=-80 \Omega \mathrm{mA}^{-1}$ and $\delta r_{\max }=20 \Omega$. During session $C: \tau=1.567 \mathrm{~s}, C$ $=0.0191 \mathrm{~V} \mathrm{~mA}^{-1}$, and $\delta v_{\max }=10 \mathrm{mV}$. (c). Four electrodes. $\epsilon=0.4$, (other data are as in Figure 3b). During session $S: K=-60 \Omega \mathrm{mA}^{-1}$, and $\delta r_{\max }=20 \Omega$. During session $C: \tau=1.57 \mathrm{~s}, C=0.00956 \mathrm{~V}$ $\mathrm{mA}^{-1}$, and $\delta v_{\max }=5 \mathrm{mV}$. Two electrodes, $\epsilon=0.2$, (other data are as in Figure 2b). During session $S: K=0 \Omega \mathrm{mA}^{-1}$ and $\delta r_{\max }=20 \Omega$. During session $C: \tau=1.61 \mathrm{~s}, C=0.0191 \mathrm{~V} \mathrm{~mA}^{-1}$, and $\delta v_{\max }=5$ $\mathrm{mV}$.

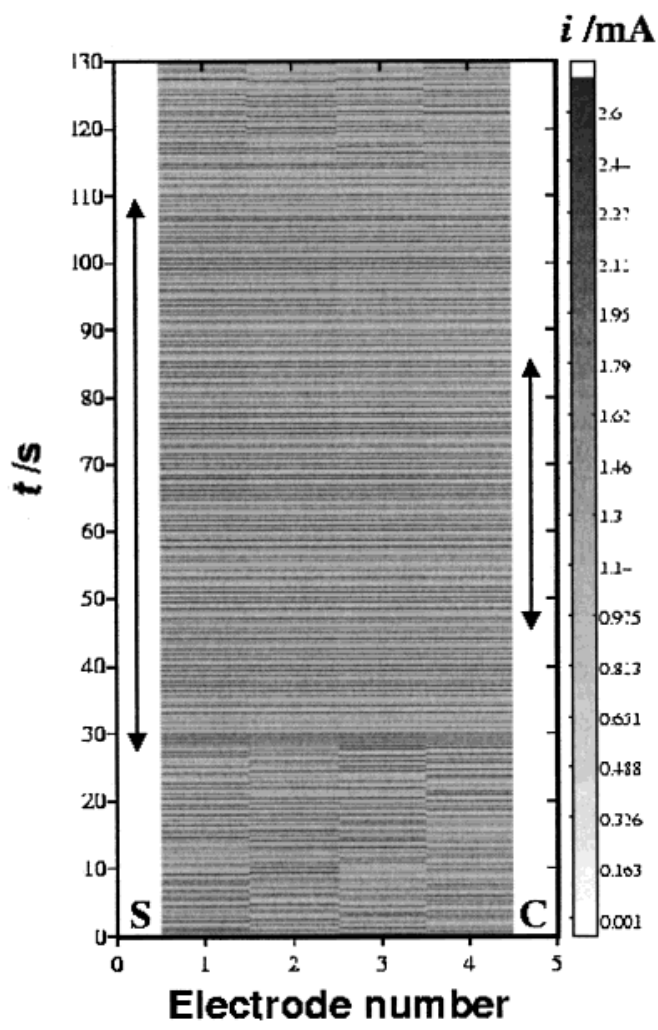

Figure 10. Grayscale plot of the individual currents of the fourelectrode system with $\epsilon=0$ (other experimental conditions are given in Figure 9a). The individual resistors are perturbed during the period of length $S$, while the circuit potential is perturbed during the period of length $C$.

\section{Concluding Remarks}

In spatially extended systems, when coupling is not strong enough to synchronize the local elements, pattern formation can be observed. Experimental control of spatiotemporal chaos is not well established. In the present study, we aimed at a task that is intermediate between an attempt to control fully spatiotemporal chaos and controlling only a single chaotic signal; viz., we investigate the control of coupled low-dimensional chaotic oscillators. Although the global dynamics were highdimensional, the system could be divided into relatively small number of low-dimensional chaotic subsystems.

The high-dimensional chaotic electrodissolution of Ni electrodes was controlled using properly chosen external feedbacks: the perturbation of the resistors connected to the electrodes and of the circuit potential. The external global coupling of the elements through the perturbation of the individual resistors resulted in a synchronized state. In addition to the synchronized chaotic state, partially synchronized patterns (clusters) were also observed using nonvanishing perturbations. Uniform periodic behaviors were then stabilized from the synchronized chaotic system using an additional control parameter (circuit potential).

In high-dimensional chaotic systems the probability of visiting the neighborhood of an unstable periodic orbit might be very small. Thus, for practical applications some target procedures may be desired so as to reduce the chaotic transient time. In some of our experiments synchronization was found to be an efficient targeting procedure.

We have applied a combination of low-dimensional techniques to control a high-dimensional chaotic process. By a lowdimensional technique we mean that the embedding dimension for data processing was less than 3. Synchronization did not 
require embedding at all, while for control the embedding dimension was only two. The price for using low-dimensional techniques to control a high-dimensional process was to use a large number of control signals. For $n$ subsystems (electrodes) we applied $n+1$ signals. Using a high-dimensional control technique could reduce the number of signals; however, that would require a larger embedding dimension (resulting in complicated calculations). Using larger number of subsystems and fewer parameters for controlling high-dimensional chaotic processes in experiments provides a challenging task for future studies.

Acknowledgment. This work has been supported by National Science Foundation (USA), the Fulbright HungarianAmerican Exchange Program and the following Hungarian Research Grants: OTKA 25375 and FKFP 0455/1997.

\section{References and Notes}

(1) Focus issue on control and synchronization of chaos: Chaos 1997, 240

(2) Petrov, V.; Gáspár, V.; Masere, J.; Showalter, K. Nature 1993, 361,

(3) Peng, B.; Petrov, V.; Showalter, K. J. Phys. Chem. 1991, 95, 4597.

(4) Pyragas, K. Phys. Lett. A 1992, 170, 421.

(5) Schneider, F. W.; Blittersdorf, R.; Förster, A.; Hauck, T.; Lebender, D.; Müller, J. J. Phys. Chem. 1993, 97, 12244.

(6) Lekebusch, A.; Förster, A.; Schneider, F. W. J. Phys. Chem. 1995, $99,681$.

(7) Rollins, R. W.; Parmananda, P.; Sherard, P. Phys. Rev. E 1993, 47, R780.

(8) Rhode, M. A.; Rollins, R. W.; Dewald, H. D. Chaos 1997, 7, 653.

(9) Parmananda, P.; Sherard, P.; Rollins, R. W.; Dewald, H. D. Phys. Rev. E 1993, 47, R3003.

(10) Kiss, I. Z.; Gáspár, V.; Nyikos, L.; Parmananda, P. J. Phys. Chem. A 1997, 101, 8668 .
(11) Parmananda, P.; Madrigal, R.; Rivera, M.; Nyikos, L.; Kiss, I. Z.; Gáspár, V. Phys. Rev. E 1999, 59, 5266.

(12) Guderian, A.; Münster, A. F.; Schneider, F. W. J. Phys. Chem. A 1998, 102, 5059 .

(13) Parmananda, P.; Rhode, M. A.; Johnson, G. A.; Rollins, R. W.; Dewald, H. D.; Markworth, A. Phys. Rev. E 1994, 49, 5007.

(14) Kuramoto, Y. Chemical Oscillations, Waves and Turbulence; Springer: Berlin, 1984.

(15) Kaneko, K. Physica D 1989, 37, 60

(16) Cross, M. C.; Hohenberg, P. C. Rev. Mod. Phys. 1993, 65, 851

(17) Hu, G.; Qu, Z. Phys. Rev. Lett. 1994, 72, 68.

(18) Parmananda, P.; Hildebrand, M.; Eiswirth, M. Phys. Rev. E 1997, $56,239$.

(19) Auerbach, D. Phys. Rev. Lett. 1994, 72, 1184

(20) Pecora, L. M.; Carrol, T. L. Phys. Rev. Lett. 1990, 64, 821.

(21) Zanette, D. H.; Mikhailov, A. S. Phys. Rev. E 1998, 57, 276. 2561.

(23) Pecora, L. M.; Carroll, T. L.; Johnson, G. A.; Mar, D. J. Chaos $1997,7,520$ 635.

24) Kocarev, L.; Tasev, Ž.; Stojanovski, T.; Parlitz, U. Chaos 1997, 7 ,

(25) Ding, M.; Ding, E.; Ditto, W. L.; Gluckman, B.; In, V.; Peng, J.; Spano, M. L.; Yang, W. Chaos 1997, 7, 644.

(26) Dolnik, M.; Epstein, I. R. Phys. Rev. E 1996, 54, 3361.

(27) Hauser, M. J. B.; Schneider, F. W. J. Chem. Phys. 1994, 100, 1058.

(28) Parmananda, P.; Jiang, Y. J. Phys. Chem. A 1998, 102, 4532.

(29) Parmananda, P. Phys. Rev. E 1997, 56, 1595.

(30) Wang, Y.; Hudson, J. L. AIChE J. 1991, 37, 1833.

(31) Fei, Z.; Kelly, R. G.; Hudson, J. L. J. Phys. Chem. 1996, 100, 18986

(32) Fei, Z.; Hudson, J. L. Ind. Eng. Chem. Res. 1998, 37, 2172.

(33) Nakabayashi, S.; Baba, R.; Shiomi, Y. Chem. Phys. Lett. 1998, 287, 632 .

(34) Franck, U. F.; Meunier, L. Z. Naturforsch. 1953, 8b, 396.

(35) Kiss, I. Z.; Wang, W.; Hudson, J. L. Phys. Chem. Chem. Phys., accepted for publication.

(36) Kiss, I. Z.; Wang, W.; Hudson, J. L. J. Phys. Chem. B 1999, 103, 11433.

(37) Wang, W.; Kiss, I. Z.; Hudson, J. L. Chaos 2000, 10, 248.

(38) Hegger, R.; Kantz, H.; Schreiber, T. Chaos 1999, 9, 413. 